\title{
Can Quality Improvement Initiatives With Organizational Changes Improve the Delivery of Asthma Care?
}

Optimal care of the patient with asthma undoubtedly requires intensive respiratory services in the in-patient setting, particularly in the initial phase. Multiple studies have shown that standardized asthma protocols implemented in the emergency department can decrease emergency department stay, admission rates, and relapse rates. ${ }^{1,2}$ Additionally, when implemented in the in-patient setting, a decrease occurs in hospital stay and $\beta$-agonist treatments, and asthma education is improved. ${ }^{3,4}$ Despite this apparent benefit from standardized asthma protocols, only a fraction of units utilize asthma protocols routinely in clinical practice. .,6 $^{5}$

In this issue of ResPiratory CARE, Cockerham et $\mathrm{al}^{7}$ report the analysis of a quality improvement initiative to reduce the time interval between bronchodilator therapies during transitions of care from the emergency department to the medical ward in pediatric subjects with acute asthma exacerbations. They initially conducted a retrospective 15month pre-intervention analysis of the baseline time between the final emergency department respiratory assessment/administration of bronchodilator therapy and the first medical ward assessment/administration of bronchodilator therapy. The quality improvement intervention consisted of an organizational change that comprised an implementation of a physician-ordered, respiratory therapist (RT)driven asthma protocol to include respiratory assessment every $2 \mathrm{~h}$ with bronchodilator therapy provided every $4 \mathrm{~h}$ as scheduled and every $2 \mathrm{~h}$ as needed. Clinical data were collected monthly in the 15 -month period following implementation. The main finding of this study was that the use of an RT-driven asthma protocol reduced the time interval between the final emergency department respiratory assessment and medical ward respiratory assessment by approximately $20 \%$. There was no difference in hospital stay between the pre- and post-intervention period. It is likely that patient/family satisfaction was improved.

Can organizational changes consistently improve the delivery of care in asthma? Despite the availability of evidence-based guidelines for the management of asthma,

The authors have disclosed no conflicts of interest.

Correspondence: Diego J Maselli MD, 7400 Merton Minter MC111E, San Antonio, Texas 78229. E-mail: masellicacer@uthscsa.edu.

DOI: $10.4187 /$ respcare.05311 there are still gaps in the delivery of care for best outcomes. For these reasons, quality improvement initiatives have been implemented to identify areas where delivery of care is not optimal and to create plans of action to correct these deficiencies. An organizational change intervention is a type of quality improvement initiative designed to improve processes and outcomes by augmenting the level

See the Original Study on Page 1573

of care that patients receive. ${ }^{3}$ In the treatment of asthma, this can be accomplished by allocating resources to asthma programs, such as involving asthma educators, pharmacists, RTs, or other specialized caregivers with direct involvement in asthma care, and pre-establishing metrics to evaluate outcomes. As expected, these organizational changes are associated with increased costs. ${ }^{8}$ The results of the implementation of these measures have been mixed. A study by Shelledy et $\mathrm{al}^{9}$ evaluated an in-home asthma management program provided by RTs or nurses and usual care. Subjects who received augmented asthma care at home by RTs or nurses had a reduction in exacerbation rates and improvement in quality of life compared with usual care. Importantly, it was demonstrated that RT-driven programs were effective in improving asthma outcomes and costs. ${ }^{9,10}$ In patients with frequent asthma exacerbations, it has been shown that nurse-directed interventions during admissions are associated with subsequent improvements in readmission rates and decreases in loss of work or school days compared with usual care. ${ }^{11}$ Unfortunately, increasing resources toward asthma education and care is not always associated with improved outcomes. A study that evaluated the involvement of asthma educators during an emergency department visit showed that an asthma education program was associated with similar rates of exacerbations compared with usual care. ${ }^{12}$ This study illustrates the unique difficulties subjects with asthma experience during an emergency department visit, such as a disconnect between the primary care physician and the emergency department and the distractors of an acute illness on the subject and caregivers. ${ }^{13}$ Although other studies have shown benefit in this patient population with regards to subsequent emergency department visits and hospitalizations for 


\section{EDITORIALS}

asthma, there remains uncertainty as to the effect on other outcomes, such as lung function and quality of life. ${ }^{14}$

In the study by Cockerham et al, ${ }^{7}$ organizational changes that increased the frequency of evaluation of the subjects in the emergency department resulted in an increased delivery of treatment, suggesting that subjects received an appropriate therapy that otherwise would have possibly been lacking. Interestingly, this increased delivery of therapy did not translate into improved lengths of stay. It is plausible that increased bronchodilation therapy was not a major determinant for the resolution of an asthma exacerbation in this group of subjects. This is supported by studies that have shown that intermittent and continuous albuterol nebulization are equivalent for the treatment of asthma in the acute setting. ${ }^{15}$ Even though the results of this study are thought provoking, there are some limitations that deserve mention. ${ }^{7}$ The study was retrospective and therefore has the inherent pitfalls of this particular study design. Even though a pre-intervention analysis was performed, a control arm of subjects receiving usual care would have provided more robust conclusions regarding the intervention that was explored. Additionally, outcomes, such as asthma severity, subsequent asthma exacerbations, and cost, were not explored. Finally, this study represents the experience of a single site, and thus the results may not apply to other hospital systems where different asthma protocols may be in place. Nevertheless, this study exemplifies how a problem was identified in the delivery of asthma care and strategies that were used to approach it.

Quality improvement initiatives remain an important part of the continuous efforts to provide better delivery of care to patients with asthma. Although these efforts are not always linked to improved outcomes, they continue to provide insight into the difficulties that patients, caregivers, and providers face with this chronic disease. With the ongoing development of new asthma protocols and increased use of technology, it is expected that the care for patients with asthma will continue to improve. As health-care expenditures continue to skyrocket, the need for efficient health-care delivery is more important than ever. Future, prospective, randomized studies will help elucidate which organizational changes are most effective with regards to asthma outcomes and reduce the misallocation of respiratory resources thereby reducing overall costs.

\section{Sheila A Habib MD Diego J Maselli MD}

Division of Pulmonary Diseases and Critical Care University of Texas Health Science Center at San Antonio San Antonio, Texas

\section{REFERENCES}

1. Minai BA, Martin JE, Cohn RC. Results of a physician and respiratory therapist collaborative effort to improve long-term metereddose inhaler technique in a pediatric asthma clinic. Respir Care 2004;49(6):600-605.

2. Colice GL, Carnathan B, Sung J, Paramore LC. A respiratory therapist-directed protocol for managing inpatients with asthma and COPD incorporating a long-acting bronchodilator. J Asthma 2005; 42(1):29-34.

3. Bravata DM, Sundaram V, Lewis R, Gienger A, Gould MK, McDonald KM, et al. Closing the quality gap: a critical analysis of quality improvement strategies. Volume 5: Asthma care. Rockville (MD): Agency for Healthcare Research and Quality (US); 2007. Report No.: 04(07)-0051-5.

4. Kallstrom TJ, Myers TR. Asthma disease management and the respiratory therapist. Respir Care 2008;53(6):770-777.

5. Hodgson D, Rudkin SE, Oman JA, Fisher J. The frequency of reevaluation or peak flow meter documentation in acute asthma exacerbations in the emergency department: are we treating in accordance with NIH/NAEPP guidelines? Cal J Emerg Med 2005;6(3): 58-61.

6. Metcalf AY, Stoller JK, Fry TD, Habermann M. Patterns and factors associated with respiratory care protocol use. Respir Care 2015; 60(5):636-643.

7. Cockerham JR, Lowe GR, Willis R, Stecks RM, Berlinski A. Quality improvement project to improve timeliness between bronchodilator treatments from emergency department to medical wards. Respir Care 2016;61(12):XXX-XXX.

8. Sullivan SD, Lee TA, Blough DK, Finkelstein JA, Lozano P, Inui TS, et al. A multisite randomized trial of the effects of physician education and organizational change in chronic asthma care: costeffectiveness analysis of the Pediatric Asthma Care Patient Outcomes Research Team II (PAC-PORT II). Arch Pediatr Adolesc Med 2005;159(5):428-434.

9. Shelledy DC, Legrand TS, Gardner DD, Peters JI. A randomized, controlled study to evaluate the role of an in-home asthma disease management program provided by respiratory therapists in improving outcomes and reducing the cost of care. J Asthma 2009;46(2): 194-201.

10. Shelledy DC, McCormick SR, LeGrand TS, Cardenas J, Peters JI. The effect of a pediatric asthma management program provided by respiratory therapists on patient outcomes and cost. Heart Lung 2005; 34(6):423-428.

11. Castro M, Zimmermann NA, Crocker S, Bradley J, Leven C, Schechtman KB. Asthma intervention program prevents readmissions in high healthcare users. Am J Respir Crit Care Med 2003;168(9):10951099.

12. Brown MD, Reeves MJ, Meyerson K, Korzeniewski SJ. Randomized trial of a comprehensive asthma education program after an emergency department visit. Ann Allergy Asthma Immunol 2006; 97(1):44-51.

13. Zorc JJ, Scarfone RJ, Li Y, Hong T, Harmelin M, Grunstein L, Andre JB. Scheduled follow-up after a pediatric emergency department visit for asthma: a randomized trial. Pediatrics 2003;111(3): 495-502.

14. Boyd M, Lasserson TJ, McKean MC, Gibson PG, Ducharme FM, Haby M. Interventions for educating children who are at risk of asthma-related emergency department attendance. Cochrane Database Syst Rev 2009;(2):CD001290.

15. Rodrigo GJ, Rodrigo C. Continuous vs intermittent beta-agonists in the treatment of acute adult asthma: a systematic review with metaanalysis. Chest 2002;122(1):160-165. 